\title{
SEGURANÇA NA IDENTIFICAÇÃO DO PACIENTE: A REALIDADE DE UMA UNIDADE PSIQUIÁTRICA
}

\section{PATIENT IDENTIFICATION SAFETY: THE REALITY OF A PSYCHIATRIC UNIT}

\section{SEGURIDAD DE IDENTIFICACIÓN DEL PACIENTE: LA REALIDAD DE UNA UNIDAD PSIQUIÁTRICA}

Andreia Guerra Siman ${ }^{1}$, Pâmela Andrade Lucarelli2, Marilane de Oliveira Fani Amaro ${ }^{3}$, Fernanda Batista Oliveira Santos ${ }^{4}$, Simone Graziele Silva Cunha ${ }^{5}$

\section{RESUMO}

Objetivo: Identificar as ações realizadas pela equipe de enfermagem, para alcançar a meta de segurança de identificação correta de pacientes, em uma unidade de internação psiquiátrica. Método: Estudo de caso, com abordagem qualitativa, realizado em uma unidade psiquiátrica de um hospital de ensino. A coleta de dados ocorreu mediante observação e entrevistas, com roteiro semiestruturado, com a equipe de enfermagem, e analisadas segundo a análise de conteúdo. Resultados: a identificação ocorria, por meio da admissão, uso de placas de identificação no leito, identificação no prontuário e uso de quadro branco no posto de enfermagem. $O$ envolvimento da família melhora a identificação e a segurança do paciente, mas não houve identificação de uma prática baseada nos protocolos nem atuação do núcleo de segurança do paciente. Conclusão: Não havia uma sistematização no processo de identificação na unidade. Em razão das singularidades do pacientes psiquiátricos urge a necessidade de melhorias e identificação adequada.

Descritores: Segurança do Paciente; Sistemas de Identificação de Pacientes; Transtornos Mentais.

\begin{abstract}
Objective: To identify actions taken by the nursing staff to achieve the safety goal of correctly identifying patients in a psychiatric inpatient unit. Method: Case study with a qualitative approach, conducted in a psychiatric unit of a teaching hospital. Data collection occurred through observation and interviews, using a semi-structured script, with the nursing staff, and analyzed according to content analysis. Results: identification occurs via admission; use of identification plates in the bed; identification in the medical records; and use of whiteboard in the nursing station. Family involvement improves patient identification and safety, but there was no identification of a protocol-based practice or performance of the patient safety core. Conclusion: There was no systematization in the identification process in the unit. Due to the uniqueness of the psychiatric patient, there is a need for improvement and proper identification.
\end{abstract}

Descriptors: Patient Safety; Patient Identification Systems; Mental Disorders.

\section{RESUMEN}

Objetivo: identificar las acciones tomadas por el personal de enfermería para lograr el objetivo de tener la seguridad en identificar correctamente a los pacientes en una unidad de hospitalización psiquiátrica. Método: estudio de caso con enfoque cualitativo, realizado en una unidad psiquiátrica de un hospital universitario. La recopilación de datos se realizó mediante observación y entrevistas, con un guión semiestructurado, con el personal de enfermería, y se analizaron de acuerdo con el análisis de contenido. Resultados: la identificación se produjo a través de la admisión, el uso de placas de identificación en la cama, la identificación en los registros médicos y el uso de pizarra en la estación de enfermería. La participación familiar mejora la identificación y la seguridad del paciente, pero no se identificó una práctica basada en el protocolo o el desempeño del núcleo de seguridad del paciente. Conclusión: no hubo sistematización en el proceso de identificación en la unidad. Debido a la singularidad del paciente psiquiátrico, existe una necesidad de mejora e identificación adecuada.

Descriptores: Seguridad del Paciente; Sistemas de Identificación de Pacientes; Trastornos Mentales.

${ }^{1}$ Enfermeira. Doutora em enfermagem. Departamento de Medicina e Enfermagem, Universidade Federal de Viçosa. ${ }^{2}$ Enfermeira. Graduação em Enfermagem pela Universidade Federal de Viçosa. ${ }^{3}$ Enfermeira. Doutora. Departamento de Medicina e Enfermagem. Universidade Federal de Viçosa. ${ }^{4}$ Enfermeira. Doutora em Enfermagem. Departamento de Enfermagem Básica. Universidade Federal de Minas Gerais. ${ }^{5}$ Enfermeira. Doutoranda em Enfermagem Escola de Enfermagem UFMG. Docente na UEMG. DOI: 


\section{INTRODUÇÃO}

A segurança do paciente é um assunto crescente em discussão, no âmbito mundial, pleiteada, principalmente, pelos serviços de saúde .A fim de reduzir os erros e ampliar o número de práticas seguras, as instituições de saúde estão investindo em ações que primem pela qualidade assistencial e busquem a difusão de uma cultura de segurança aos pacientes, profissionais e ambiente ${ }^{(1-2)}$.

A essência da segurança do paciente é caracterizada pela preocupação com a significância da ocorrência de eventos adversos (EA), isto é, com lesões ou danos ao paciente ocasionados pelo cuidado em saúde ${ }^{(1)}$.Sabe-se que a ocorrência dos eventos, envolve custos sociais, econômicos pertinentes e numerosos, podendo implicar em danos inconvertíveis aos pacientes e familiares ${ }^{(1,3)}$.

Nesse contexto, a Organização Mundial da Saúde (OMS) mobilizou-se frente às questões de práticas seguras e da qualidade do cuidado em saúde e, em 2004, foi criada a Aliança Mundial para a Segurança do Paciente, que se voltou para a criação e o desenvolvimento de políticas em prol da segurança do paciente para todos os países membros da Organização Mundial de Saúde (OMS) $)^{(4)}$.

Em 2013, foi criado, no Brasil, o Programa Nacional de Segurança do Paciente (PNSP), estabelecendo protocolos para atendimento às metas internacionais de segurança e determinando a criação de Núcleos de Segurança do Paciente (NSP), nos serviços de saúde. Essas mudanças estão focadas nas seis metas de segurança, preconizadas pela OMS, tendo, como meta número um, a correta identificação do paciente, processo pelo qual assegura ao paciente os cuidados, procedimentos e/ou tratamentos, prevenindo erros e riscos para a segurança $a^{(4-5)}$.

Assim, entende-se que, para impedir o erro, é preciso reestruturar o sistema de atendimento hospitalar, reconhecendo que nenhuma instituição está inatingível ao problema de identificação do paciente ${ }^{(6)}$.

Para o paciente psiquiátrico, a internação hospitalar é uma modalidade de tratamento especializada, utilizada com mais frequência, quando os pacientes são considerados perigosos para si ou para outros, ou que necessitam de serviços intensivos. Os objetivos do tratamento hospitalar são específicos e focados na estabilização de comportamentos, abordando a crise que precipitou a hospitalização e alinhamento dos serviços para que o paciente retorne à sua comunidade ${ }^{(7)}$.

Para tentar garantir a segurança do paciente, durante a internação, existem algumas estratégias para a correta identificação do paciente como, enfatizar que todos os profissionais de saúde sejam responsáveis pela sua implementação e confirmação da ação. $O$ paciente e familiares, por sua vez, devem estar envolvidos ativamente no processo e receber informações sobre a importância da correta identificação. Na admissão e na prestação de cuidado, deve se usar, pelo menos, dois identificadores para fazer essa identificação, assim como, padronizar as abordagens de identificação do paciente entre as diversas unidades e instituições, dentro de um sistema de saúde ${ }^{(6,8)}$.

Assegurar a correta identificação do paciente, mesmo que o profissional esteja familiarizado com ele, garante o direito de o paciente receber o cuidado correto ${ }^{(6,8)}$.

Para este estudo, parte-se do pressuposto de que uma identificação eficaz envolve fornecer a identificação do paciente, desde o momento de sua admissão, possuir um método visual e automatizado de vincular o paciente à sua documentação médica-terapêutica e minimizar a possibilidade dos dados de identificação serem transferidos a uma outra pessoa ${ }^{(6)}$.

Neste contexto, a correta identificação do paciente psiquiátrico torna-se relevante subsídio para uma prática mais segura em decorrência das particularidades do paciente. A unidade de saúde mental representa um cenário assistencial complexo, envolvendo paciente com agressividade física e verbal, agitação extrema, episódios de delírios, psicose aguda e confusão mental ${ }^{(9)}$.Ademais, poucos pesquisadores de serviços de saúde estudam psiquiatria hospitalar, e a questão não tem sido uma prioridade entre financiadores de pesquisa. Várias alavancas políticas poderiam começar a resolver essas deficiências, o que inclui alinhar os estímulos ao atendimento centrado no paciente, realizar análises de incidentes nessa área e incentivo de mais pesquisas ${ }^{(10)}$.

O paciente psiquiátrico necessita de uma assistência de enfermagem contínua, de observação constante, considerando os episódios de humor instável, tentativas de fuga e suicídio. Identificar as necessidades do paciente psiquiátrico permite $\mathrm{o}$ estabelecimento de 
intervenções e tratamentos individualizados, facilitando e efetivando a reabilitação ${ }^{(11)}$. A correta identificação, como uma das metas de segurança, se torna indispensável e essencial no resultado final: uma assistência segura e de qualidade.

Por considerar a importância da temática e perceber a existência de fragilidades, optou-se por aprofundar o olhar na identificação correta dos pacientes, em um setor de internação de pacientes psiquiátricos de um hospital de ensino.

Com as considerações apresentadas, surgiram as questões norteadoras desta pesquisa: Como a equipe de enfermagem tem identificado os pacientes da unidade psiquiátrica? Como a equipe tem reduzido os danos e promovido a segurança do paciente nesse setor? Dessa maneira, no presente estudo, tem-se como objetivo identificar as ações realizadas pela equipe de enfermagem, para alcançar a meta de segurança de identificação correta de pacientes em uma unidade de internação psiquiátrica.

\section{METÓDOS}

Trata-se de um estudo de caso qualitativo, que tem por objetivo analisar uma unidade social, buscando entender os fenômenos sociais complexos e responder "como" e "por que" eles ocorrem. Esse método de pesquisa foi escolhido, pois permite o pesquisador captar características significativas presentes em contexto real ${ }^{(12)}$.

A unidade de análise foi o serviço de psiquiatria de um hospital de médio porte, filantrópico e de ensino, vinculado à uma universidade federal, localizada na Zona da Mata de Minas Gerais, Brasil. O hospital é participante da Rede de Hospitais Sentinela, e, portanto, que possui comissão de gerenciamento de riscos e NSP desde 2013.

O serviço de psiquiatria é uma unidade de internação com quinze leitos e é referência no atendimento de uma microrregião composta por nove municípios... serviço possuía uma equipe de enfermagem composta por dez profissionais, sendo dois enfermeiros e oito técnicos de enfermagem (TE)(plantão diurno e noturno), com escala de trabalho de $12 \times 36 \mathrm{~h}$, dois TE por plantão, e um enfermeiro de oito horas diárias e outro de seis horas diárias. Ressalta-se que é a equipe de enfermagem que realiza a identificação do paciente no serviço.

Os participantes da pesquisa foram eleitos com os critérios de inclusão: profissional da equipe de enfermagem (enfermeiro ou TE) da unidade de internação psiquiátrica, independentemente do tempo de exercício na função. Utilizou-se como critérios de exclusão :enfermeiros ou TE afastados do cargo por qualquer motivo, no período da coleta de dados. Portanto, foi entrevistada a totalidade da equipe: dois enfermeiros e oito TE.

A coleta de dados se deu por meio de entrevistas e observação. Para as entrevistas, utilizou-se um roteiro semiestruturado, elaborado pelos pesquisadores e realizado teste piloto, sem necessidades de alterações. A observação se deu antes e após as entrevistas, até alcançar o objetivo proposto, com anotações em um diário de campo. A equipe da psiquiatria foi observada por 32 horas. A coleta se deu no período de julho a agosto de 2016. As entrevistas foram realizadas pela pesquisadora principal e tiveram duração média de 20 minutos, realizadas no próprio local de trabalho, em um lugar reservado e confortável para que os participantes expusessem suas ideias e houve agendamento prévio com cada um. O roteiro abordava questões sobre a importância da identificação do paciente; as ações e estratégias realizadas na identificação, tipos de treinamento para melhorar a identificação do paciente, ocorrência de erros e EA e a atuação do NSP no setor.

As entrevistas foram áudio gravadas com anuência dos profissionais e os registros foram transcritos na íntegra e, após essa etapa, passaram por processo analítico e descritivo, a partir do referencial da Análise de Conteúdo ${ }^{(11)}$.Para garantir $\mathrm{o}$ anonimato dos participantes, os depoimentos foram codificados com a letra $P$ (participante) seguida de numeral arábico, conforme a ordem em que foram entrevistados.

Para a Análise de Conteúdo foi realizada uma sequência cronológica das seguintes etapas: pré-análise, exploração do material e tratamento dos resultados, inferência e interpretação ${ }^{(13)}$. Assim, inicialmente foi realizada uma leitura flutuante e exaustiva das questões das entrevistas de forma a haver uma familiarização com o texto e obter uma compreensão sobre o que o sujeito buscava transmitir. Em seguida, procedeu-se a seleção temática, que consistiu em identificar os núcleos de sentido, ou elementos semanticamente semelhantes, para posterior categorização e interpretação à luz da literatura.

$O$ estudo foi conduzido segundo as normas da Resolução 466/2012 do Conselho Nacional de Saúde, iniciando-se após apreciação e aprovação 
do Comitê de Ética em Pesquisa com Seres Humanos da Universidade Federal de Viçosa (CAAE: 44109015.0.0000.5149). Todos os participantes assinaram o Termo de Consentimento Livre e Esclarecido.

\section{RESULTADOS E DISCUSSÃO}

Participaram do estudo 10 profissionais: $56 \%$ homens e $44 \%$ mulheres. Acredita-se que é uma característica do serviço de psiquiatria admitir mais homens a mulheres, em razão de características do paciente em sofrimento mental. A faixa etária era de 30 a 45 anos, e a média de tempo de formação foi de 8 anos, com tempo máximo de 19 anos. Os enfermeiros não tinham especialização na área.

Da análise resultaram as seguintes categorias: Ações realizadas na identificação do paciente psiquiátrico; Envolvimento da família na identificação e segurança do paciente; Identificação do paciente: dificuldades identificadas.

Para facilitar a compreensão da análise, no Quadro 1, sumarizam-se as categorias, subcategorias e alguns depoimentos dos participantes sobre a identificação do paciente na unidade psiquiátrica.

Quadro1 - Categorias e subcategorias: ações realizadas para alcançar a meta de segurança de identificação correta de pacientes em uma unidade de internação psiquiátrica.

\begin{tabular}{|c|c|c|}
\hline Categoria & Subcategorias & Depoimentos \\
\hline \multirow[t]{2}{*}{$\begin{array}{l}\text { 1-Ações realizadas na identificação do } \\
\text { paciente psiquiátrico }\end{array}$} & Identificação no leito & $\begin{array}{l}\text { "Sempre antes de realizar qualquer } \\
\text { procedimento com o paciente eu o } \\
\text { chamo pelo nome e verifico o número } \\
\text { do leito. Ter a placa de identificação na } \\
\text { cama ajuda muito".(P2) } \\
\text { “A primeira coisa que eu pego é o } \\
\text { prontuário do paciente, onde olho o } \\
\text { nome, e já coloco no leito do paciente o } \\
\text { papel identificando". (P4) } \\
\text { A identificação do paciente é muito } \\
\text { precária. Lá é identificado no material, o } \\
\text { nome do paciente vai no material, } \\
\text { medicação... Estão pensando em adotar } \\
\text { as pulseiras e existe a identificação do } \\
\text { paciente no leito. (P10) } \\
\text { Aqui tem muito risco de queda, colocar } \\
\text { dois Sebastiões dentro do mesmo } \\
\text { quarto. Um Sebastião do lado do outro. } \\
\text { O enfermeiro até tenta gerenciar, mas } \\
\text { saiu vaga, só tem aquela vaga masculina, } \\
\text { então, coloca aquele paciente lá, ai } \\
\text { depois deveria realocar. A identificação } \\
\text { do paciente é muito pouco, só coloca ID } \\
\text { do paciente no leito. (P8) }\end{array}$ \\
\hline & Quadro de identificação & $\begin{array}{l}\text { "Sabemos quem é o paciente por causa } \\
\text { desse quadro que fica no posto de } \\
\text { enfermagem".(P3) } \\
\text { "Sempre identifico pelo número do leito } \\
\text { e pelo nome do paciente".(P7) }\end{array}$ \\
\hline $\begin{array}{l}\text { 2-Envolvimento da família na } \\
\text { identificação e segurança do paciente }\end{array}$ & Admissão com familiares & $\begin{array}{l}\text { “Na admissão, os familiares participam } \\
\text { do processo por meio de perguntas e } \\
\text { logo em seguida são liberados".(P1) } \\
\text { “Na admissão, costumo conversar com } \\
\text { os familiares e às vezes com o paciente, } \\
\text { depende da complexidade do paciente. } \\
\text { A família tem que participar". (P6) }\end{array}$ \\
\hline
\end{tabular}

"continua na página seguinte" 


\begin{tabular}{|c|c|c|}
\hline Categoria & Subcategorias & Depoimentos \\
\hline \multirow[t]{4}{*}{$\begin{array}{l}\text { 3-Identificação do paciente: dificuldades } \\
\text { identificadas }\end{array}$} & Falta de sistematização no processo & $\begin{array}{l}\text { "Quando encontramos duas pessoas } \\
\text { com o mesmo nome, não temos } \\
\text { dificuldade, por que temos poucos } \\
\text { leitos, e quando acontece de ter dois } \\
\text { com nomes iguais, avaliamos a diferença } \\
\text { pela fisionomia deles mesmo, cada um é } \\
\text { de um jeito".(P5) }\end{array}$ \\
\hline & $\begin{array}{l}\text { Singularidades ao paciente com } \\
\text { transtorno mental }\end{array}$ & $\begin{array}{c}\text { "Na psiquiatria, os pacientes sempre } \\
\text { tiram e rasgam os papeis colocados, mas } \\
\text { ficamos sempre atento para saber quem } \\
\text { é quem, e não cometer nenhum erro". } \\
\text { (P4) } \\
\text { "Se eu for só pela numeração do leito, } \\
\text { eu faço a medicação errada, porque eles } \\
\text { trocam de leitos se estiverem delirando" } \\
\text { (P7) } \\
\text { “Na psiquiatria, cada caso é um caso. } \\
\text { Temos paciente com transtornos, } \\
\text { pacientes usuários de drogas, tem } \\
\text { pessoas que estão ali por causa do } \\
\text { alcoolismo, vários motivos, e isso acaba } \\
\text { dificultando a identificação".(P8) }\end{array}$ \\
\hline & $\begin{array}{l}\text { Falta atuação do núcleo de segurança do } \\
\text { paciente }\end{array}$ & $\begin{array}{l}\text { “Nesses meses que estou aqui, nunca } \\
\text { ouvi falar de comissão. Agora a ética de } \\
\text { enfermagem, talvez essa comissão possa } \\
\text { estar atuando na segurança do } \\
\text { paciente". (P1) } \\
\text { “Olha, para ser sincero eu nem sei o que } \\
\text { faz esse núcleo, acho que aqui não } \\
\text { existe”. (P9) } \\
\text { “Bem difícil; mas que tipo de comissão e } \\
\text { núcleo é esse? O paciente é seguro o } \\
\text { tempo inteiro, quem não tem segurança } \\
\text { somos nós, pois eles podem nos bater. } \\
\text { Temos que conversar com eles, e ser } \\
\text { amiga deles".(P4) } \\
\text { "Temos comissão sim, só não sei como } \\
\text { funciona, mas sempre fica pregado no } \\
\text { mural, a gente passa e lê".(P5) }\end{array}$ \\
\hline & Treinamentos & $\begin{array}{l}\text { "Nunca tive nenhum treinamento de } \\
\text { como identificar os pacientes". (P1) } \\
\text { "Estou no hospital a muito tempo e não } \\
\text { tive esse momento de aprender a } \\
\text { identificar o paciente". (P7). } \\
\text { "O enfermeiro sempre fala que tem que } \\
\text { identificar, que temos que prestar a } \\
\text { atenção. Ficamos perdidos, a instituição } \\
\text { precisa oferecer um treinamento para } \\
\text { identificar o paciente".(P4) } \\
\text { "A gente está sempre orientando, faz } \\
\text { educação continuada, a gente faz as } \\
\text { reuniões, mas a gente não trabalha em } \\
\text { cima de penalidades, a gente trabalha } \\
\text { em cima de orientações, da educação". } \\
\text { (P10) }\end{array}$ \\
\hline
\end{tabular}

Fonte: Banco de dados, 2016.

Os resultados apontam que as ações desenvolvidas pela equipe de enfermagem, na unidade psiquiátrica, englobavam a identificação na beira do leito, preenchida na admissão; preenchimento de um quadro branco, contendo nome completo dos pacientes e leito, localizado no posto de enfermagem, com o objetivo de diminuir futuros erros e agilizar o processo de trabalho da equipe. Porém, em razão de características do paciente com transtorno mental, os achados da observação e os 
depoimentos apontaram que nem sempre essa identificação era mantida na beira do leito.

Na prática do cuidado em saúde, ainda há uma demora no desenvolvimento de esforços para melhorar a segurança do paciente. Esse atraso é, especialmente, na psiquiatria hospitalar, onde há risco de danos ao paciente ${ }^{(10)}$. Os resultados do presente estudo, corroboram com a literatura, demostrando que são poucos os esforços no alcance da meta de segurança de identificação correta de pacientes, na unidade de internação psiquiátrica.

Por meio da observação, foi possível analisar as placas de identificação no leito, as quais eram preenchidas pelo técnico de enfermagem na admissão do paciente, preenchendo nome completo, registro, nome da mãe e nome do médico responsável. Além da avaliação do risco de queda, risco de lesão e alergias. Apesar do instrumento, várias vezes 0 paciente não tinha a folha na cabeceira do leito e a equipe alegava que o paciente havia retirado.

Em relação ao quadro de identificação, ele ficava localizado no posto de enfermagem, e identificava nome, motivo, data de internação e médico responsável. Era preenchido e conferido no início do plantão pelo enfermeiro e atualizado, ao longo do dia, com as altas e admissões, pelo técnico de enfermagem. Antes da realização de cuidados e procedimentos como banho, alimentação e administração de medicamentos, foi observado que o profissional chamava $o$ paciente pelo primeiro nome. No período da coleta de dados, não houve nenhuma visita da equipe do NSP no serviço, nem foi presenciado um treinamento com a equipe.

Por vezes foram identificados prontuários com cabeçalho incompleto, no que tange a identificação do paciente, nomes incompletos em registros de enfermeiros, de técnicos de enfermagem, nos registros e prescrições médicas. Não foram identificados procedimentos operacionais padrões ou protocolos sobre a identificação correta do paciente, disponíveis no setor.

Quando o paciente era recebido no setor, proveniente de outro serviço como o CAPS,Pronto Atendimento, ou outro, não havia nenhuma estratégia para a identificação do paciente. Os familiares acompanhavam o paciente até o serviço, portando de documentos da internação e outros como admissão médica e prescrição.
A identificação do paciente deve ter como finalidade determinar com segurança a legitimidade do tratamento ou procedimento e assegurar que seja executado o que o paciente necessita $^{(2,4-5)}$. Na prática, a identificação correta do paciente, faz parte do cuidado de enfermagem e, na presente pesquisa, os resultados apontaram falhas em relação a esse processo, deixando o paciente vulnerável ao erro. Identificar o paciente apenas pelo nome e número do leito, ou ainda memorizar a fisionomia do paciente, caracterizam uma ação insegura e em desacordo com os protocolos recomendados pelo MS e PNSP, podendo interferir nas demais etapas da assistência multiprofissional, sendo uma ação primordial para garantia a qualidade e a segurança do paciente ${ }^{(2)}$.

Ressalta-se, que os depoimentos e os dados da observação apontaram que o setor não possuía uma padronização no processo de identificação do paciente. A partir do lançamento do PNSP, as instituições necessitam compatibilizar os dispositivos preconizados pelo protocolo e o desejo dos pacientes ${ }^{(2)}$.Assim, a administração da instituição, juntamente com outros líderes, devem investir na identificação dos pacientes, fazendo uso de tecnologias favoráveis, disponíveis e comprovadas como estratégias na prevenção do erro, tais como: pulseiras, etiquetas digitalizadas, rótulos e códigos de barra, fazendo assim com que os profissionais disponham de recursos adequados na realização dessa prática ${ }^{(6)}$.

As diretrizes de implantação da meta de identificação correta dos pacientes recomendam que as instituições adotem métodos uniformes para identificar seus pacientes, como pulseiras identificação, com o mínimo de dois elementos identificadores e também assegurar a sua verificação antes dos procedimentos de maior risco, como antes da administração de medicamentos, de sangue e hemocomponentes, coleta de amostras, exames diagnósticos e procedimentos cirúrgicos ${ }^{(14)}$.

Os resultados apresentados também demonstraram que a estratégia da identificação na beira do leito, na ala psiquiátrica, não tem sido eficaz, nesse sentido, estudos apontam que, além das placas, nas cabeceiras, podem ser utilizados adesivos nas roupas e para certificar-se de que os pacientes sejam identificados de forma correta $^{(14)}$. A identificação do paciente deve ocorrer como um processo sistematizado e 
atentar para critérios como integridade e legibilidade ${ }^{(2)}$.

Para maior segurança, a identificação de todos os pacientes deve ser realizada em sua admissão no serviço e deve permanecer durante todo o tempo em que paciente estiver submetido ao cuidado. Ressalta-se que os serviços de saúde devem desenvolver, implementar e revisar regularmente processos que facilitem a correta identificação dos pacientes, inclusive na passagem de caso entre as equipes de saúde, na transferência e na alta do paciente ${ }^{(8)}$.

A respeito da segunda categoria, os participantes relataram sobre a importância do envolvimento da família, no processo de identificação do paciente, sendo peça fundamental, principalmente, na admissão efetiva do paciente, auxiliando na informação de dados ao entrar na unidade. A família, no contexto do paciente psiquiátrico, auxilia na confirmação da identidade dos pacientes que deve ser realizada antes de qualquer cuidado, incluindo a administração de medicamentos ${ }^{(15)}$, assim como, da coleta de material para exame, da entrega da dieta e da realização de procedimentos invasivos.

Envolver a família/acompanhante e explicar o propósito de identificar o paciente facilita na prestação dos cuidados a serem realizados, e além disso, o envolvimento de todos faz parte dos esforços para melhorar a segurança ${ }^{(10)}$.O acompanhamento e o vínculo da família com o paciente psiquiátrico são fundamentais para a compreensão e terapia, durante a internação, dando apoio, carinho, dedicação e auxiliando na prevenção de erros no período da internação ${ }^{(16)}$.

A família é um suporte básico para a vida de qualquer pessoa, mas para os pacientes psiquiátricos, se torna ainda mais essencial pelo fato de necessitarem dos cuidados e acompanhamento fora do ambiente hospitalar. Desse modo, é um importante grupo social, e a equipe de enfermagem e saúde devem auxiliar na interação e fortalecimento das relações ${ }^{(14)}$.

$\mathrm{Na}$ categoria Identificação do paciente: dificuldades identificadas, a análise ressalta falhas importantes no processo de identificação do paciente e lacunas no conhecimento da equipe quanto a segurança do paciente. A equipe de enfermagem só identificou dificuldades quando ocorrem homônimos e pacientes com quadros instáveis.
Vale ressaltar, que na unidade psiquiátrica, os pacientes apresentam singularidades relacionadas à medicação em uso, trocam de leitos entre si, rasgam as placas de identificação, quadros instáveis, necessitando de cuidados específicos às pessoas com transtorno mental. Dessa maneira, torna-se imprescindível a difusão da relevância da identificação correta nessa unidade, que se torna peculiar por se tratar de tais pacientes ${ }^{(17)}$.

Um dos participantes relatou que não havia a necessidade de identificação dos pacientes, em razão do pequeno número de leitos. No entanto, cabe ressaltar que os pacientes psiquiátricos são dotados de complexidades e atendidos por diversas equipes que, na maioria das vezes, prestam cuidados em apenas uma parte do dia (2). Assim, se a identificação estiver de forma incorreta, pode acontecer falhas e/ou erros em todo o processo do cuidado.

Pesquisas internacionais evidenciam que a alta incidência de problemas e erros estão relacionados à identificação incorreta de pacientes. Anualmente, cerca de 850 pacientes nos Estados Unidos são transfundidos com sangue destinados a outros pacientes e, aproximadamente, 3\% desses pacientes evoluem para óbito. Em cada 1.000 pacientes que recebem transfusões de sangue ou de hemocomponentes, um indivíduo recebe a destinada a outra pessoa ${ }^{(15)}$.

Especificamente, na psiquiatria, foi encontrado um estudo que evidencia a falta de utilização da pulseira de identificação do paciente, na clínica psiquiátrica, justificando o desuso pelo pequeno número de pacientes, o que facilitava memorizar os nomes dos mesmos $^{(18)}$. Nesse sentido, os erros ocorridos, geralmente, estão relacionados com o registro incorreto do nome nas folhas de admissão ou no próprio prontuário, com a frequente incidência de homônimos e com o processo inadequado de verificação do nome do paciente ${ }^{(19)}$.

Estudo internacional enfatiza que nem sempre os pacientes psiquiátricos acessam cuidados de saúde adequados e podem sofrer desigualdades nos cuidados prestados. O uso de medicamentos para tratar doenças mentais é um dos pilares da prática moderna. O tratamento envolve o uso de medicamentos de alto risco como lítio, antipsicóticos de depósito e clozapina, muito comuns, nessa população e apresentam desafios únicos para o uso seguro de 
medicamentos. Para agravar, nem todos os usuários de serviços de saúde mental podem se sentir envolvidos nas decisões sobre o tratamento medicamentoso(20). São dados que reforçam a importância de uma assistência mais segura ao paciente de transtorno mental.

Os erros de identificação podem ocorrer em praticamente todos os aspectos do diagnóstico e tratamento. Os pacientes podem estar sedados, desorientados ou não totalmente alertas; podem mudar de leitos, quartos ou setores dentro do hospital; podem ter deficiências sensoriais ou podem estar sujeitos a outras situações que possam levar a erros de identificação ${ }^{(21)}$.

Nessa categoria, também foi identificado que a equipe não reconhece a atuação do NSP no setor de psiquiatria, e os resultados apontaram desconhecimento sobre segurança do paciente e sobre as metas internacionais de segurança do paciente.

ONSP é a instância do serviço de saúde criada para promover e apoiar a implementação de ações voltadas à segurança do paciente em todos os setores da instituição, consistindo em um componente extremamente importante na busca pela qualidade das atividades desenvolvidas nos serviços de saúde. 0 paciente necessita estar seguro, independentemente do processo de cuidado a que ele está submetido. Dentre as diversas funções do NSP, se enquadra a educação permanente dos profissionais que trabalham na instituição ${ }^{(5)}$.

Os resultados ainda apontam que os profissionais não receberam treinamentos sobre como deve ser realizada a correta identificação do paciente. Por falta de uma política de educação permanente que vise à segurança do paciente, os profissionais realizam a ação de forma empírica.

Nesse sentido, a educação permanente deve caminhar junto a qualquer mudança no processo de trabalho, no sentido de aperfeiçoamento e a interação entre os sujeito, de forma a envolver toda a equipe, estimulando a atuação crítica, reflexiva, compromissada e tecnicamente eficiente, buscando superar as deformações e deficiências na formação dos trabalhadores da saúde ${ }^{(22)}$. Logo, torna-se necessário 0 envolvimento dos gestores, contribuindo de forma positiva na qualificação cotidiana de seus profissionais.

Para se alcançar êxito na correta identificação do paciente psiquiátrico, é imprescindível o envolvimento dos profissionais, comprometimento dos gestores, melhor comunicação do setor com o serviço de internação; estratégias que melhoram o processo. A utilização de indicadores de segurança do paciente e implementação de protocolos também facilitam o caminho da qualidade e segurança ${ }^{(14,23)}$.

Independente da tecnologia ou abordagem utilizada para a identificação do paciente psiquiátrico, é preciso construir um planejamento para os processos de cuidado, pois isso irá garantir a identificação adequada do paciente antes de qualquer intervenção de saúde, consequentemente, a prestação de cuidados mais seguros ${ }^{(1,6)}$.

\section{CONSIDERAÇÕES FINAIS}

$O$ estudo apresenta como a equipe de enfermagem tem realizado a identificação do paciente em um setor de internação de pessoas com transtorno mental e traz contribuições para melhorar a identificação desse paciente, tornando o cuidado mais seguro. São necessárias estratégias de identificação e uma sistematização das ações na unidade psiquiátrica. A família aparece como peça fundamental no processo melhorias de segurança do paciente.

$O$ estudo identificou que os profissionais utilizam de admissão com nome completo, placa de identificação no leito, identificação no prontuário e quadro branco no posto de enfermagem. No entanto, não há uma prática sistematizada, não há implantação de protocolos ou outras estratégias para auxiliar na correta identificação do paciente na psiquiatria, embora haja uma preocupação da equipe em reduzir os erros.

As dificuldades no processo de identificação na unidade, principalmente, em razão da complexidade e singularidades dos pacientes psiquiátricos. Entretanto, ressalta-se a importância de se realizar a identificação de forma científica e segundo as recomendações da OMS.

Os participantes não reconhecem a atuação do NSP, no setor, no entanto, é uma instância dentro da instituição que deve influir, diretamente, na educação permanente, implicando em treinamentos e, na construção de um planejamento para melhorias no alcance de práticas seguras.

Assim, o estudo tem como implicações para a prática de enfermagem, a importância do 
trabalho do enfermeiro em contribuir para o desenvolvimento de uma cultura de segurança do paciente, além de inserir estratégias de ações, para melhor identificação do paciente como pulseiras, etiquetas, implantação de Procedimento Operacional Padrão, protocolos, educação permanente, envolvimento com a equipe, para melhorar e tornar os cuidados de saúde mental mais seguros, evitando erros e eventos adversos.

Identificam-se lacunas de pesquisa que precisam ser melhor estudadas, especialmente, sobre a influência da família para o alcance de práticas mais seguras. Como limitação do estudo, observa-se que a literatura não é ampla, quando a abordagem e a identificação do paciente na psiquiatria, além de ter sido realizado em um único serviço, aspectos que dificultam comparações. Logo, o presente artigo poderá nortear outros estudos que busquem tornar o cuidado de saúde em saúde mental mais seguro.

\section{REFERÊNCIAS}

1- Siman AG, Cunha SGS, Brito MJM. The practice of reporting adverse events in a teaching hospital. Rev Esc Enferm USP. 2017;51:1-8. DOI: 10.1590/S1980-220X2016045503243

2- Hoffmeister LV, Moura GMSS. Use of identification wristbands among patients receiving inpatient treatment in a teaching hospital. Rev Latino-Am Enfermagem 2015;23(1):36-41. DOI: 10.1590/01041169.0144 .2522

3- Oliveira RM, Leitão IMTA, Silva LMS, Figueiredo SV, Sampaio RL, Gondim MM. Estratégias para promover segurança do paciente: Da identificação dos riscos às práticas baseadas em evidências. Esc Anna Nery 2014; 18(1):122-129. DOI: 10.5935/1414-8145.20140018

4- Wegner W, Silva SC, Kantorski KJC, Predebon $\mathrm{CM}$, Sanches MO, Pedro ENR. Educação para cultura da segurança do paciente: Implicações para a formação profissional. Esc Anna Nery 2016;20(3):1-8. DOI: $\quad$ 10.5935/1414$\underline{8145.20160068}$

5- Brasil, Agência de Vigilância Sanitária. implantação do núcleo de segurança do paciente em serviços de saúde. Brasília: Anvisa; 2014.

6- Porto TP, Rocha PK, Lessmann JC, Souza S, Anders JC. Identificação do paciente em unidade pediátrica: Uma questão de segurança. Rev Soc Bras Enferm Ped. 2011 [citado em 8 jun 2019]; 11(2):67-74. Disponível em: https://sobep.org.br/revista/component/zine/art icle/142-identificao-do-paciente-em-unidadepeditrica-uma-questo-de-segurana.html

7- Delaney KR. Nursing inchild psychiatric milieus: What nurses do: An update. J Child Adolesc Psychiatr Nurs. 2017;30(4):201-8. DOI: 10.1111/icap.12204

8- Brasil, Agência de Vigilância Sanitária. Assistência segura: Uma reflexão teórica aplicada à prática. Brasília: Anvisa; 2013.

9- Fried El, Borkulo CD, Cramer AO, Boschloo L, Schoevers RA, Borsboom D. Mental disorders as networks of problems: A review of recent insights. Soc Psychiatr Psychiatr Epidemiol. 2016;52(1):1-10. DOI: 10.1007/s00127-016-1319$\mathrm{z}$

10- Shields MC, Stewarte MT, Delaney KR. Patient safety in inpatient psychiatry: A remaining frontier for health policy. Health Aff. 2018;37(11):1853-61. DOI: 10.1377/hlthaff.2018.0718

11- Mukai HA, Jericó MC, Perroca MG. Care needs and workload required by institutionalised psychiatric patients. Rev Latino-Am Enfermagem 2013;21(1):1-9. DOI: 10.1590/S0104$\underline{11692013000100008}$

12- Yin RK. Estudo de caso: Planejamento e métodos. 5a ed. Porto Alegre: Bookman; 2015.

13- Bardin L. Análise de conteúdo. São Paulo: Edições 70; 2011.

14- Hemesath MP, Santos HB, Torelly EMS, Barbosa AS, Magalhães AMM. Estratégias educativas para melhorar a adesão à identificação do paciente. Rev Gaúcha Enferm. 2015;36(4):43-8. DOI: $\quad$ 10.1590/1983$\underline{1447.2015 .04 .54289}$

15- Alcântara CB, Capistrano FC, Czarnobay J, Ferreira ACZ, Brusamarello T, Maftum MA. A terapêutica medicamentosa às pessoas com transtorno mental na visão de profissionais da enfermagem. Esc Anna Nery 2018;22(2): 1-7. DOI: 10.1590/2177-9465-ean-2017-0294

16- Sousa FCP, Montenegro LC, Goveia VR, Corrêa $A R$, Rocha PK, Manzo BF. A participação da família na segurança do paciente em unidades neonatais na perspectiva do enfermeiro. Texto Contexto-Enferm. 2017;26(3):1-8. DOI: 10.1590/0104-07072017001180016

17- Prado MF, Sá MC, Miranda L. O paciente com transtorno mental grave no hospital geral: Uma revisão bibliográfica. Saúde Debate 2015;39(nesp):320-37. DOI: 10.5935/01031104.20155005419

18- Miasso Al, Cassiani SHB. Erros in the administration of medications: The scientific 
knowledge and the identification of the patient as an important aspects. Rev Esc Enferm USP 2000;34(1):16-25. DOI: $10.1590 / 50080-$ $\underline{62342000000100003}$

19- Tase TH, Quadrado ERS, Tronchin DMR. Evaluation of the risk of misidentification of women in a public maternity hospital. Rev Bras Enferm. 2018;71(1):120-5. DOI: 10.1590/00347167-2017-0134

20- Keers RN, Plácido M, Bennett K, Clayton K, Brown $P$, Ashcroft DM. What causes medication administration errors in a mental health hospital? A qualitative study with nursing staff. PLoS One 2018;13(10):e0206233. DOI: 10.1371/journal.pone.0206233.eCollection 2018

21- Wegner W, Silva SC, Kantorski KJC, Predebon $\mathrm{CM}$, Sanches MO, Pedro ENR. Educação para cultura da segurança do paciente: Implicações para a formação profissional. Esc Anna Nery 2016;20(3):1-8. DOI: $\quad$ 10.5935/1414$\underline{8145.20160068}$

22- Almeida TMC, Santos RMM, Sampaio DMN, Vilela ABA. Planejamento e desenvolvimento de ações de Educação Permanente em Saúde na perspectiva do PMAQ-AB. Saúde Debate 2019;43(nesp 1):77-85. DOI: 10.1590/0103$\underline{11042019 s 106}$

23- Fujii Neta A, Girardi C, Santos DTR, Oliveira JLC, Oliveira RP, Maraschin MS, et al. Adesão à identificação do paciente em hospital universitário público. Rev Adm Saúde 2018;70(18):2-13. DOI: $10.23973 /$ ras.70.70

Note: Estudo proveniente de uma pesquisa maior intitulada 'Práticas cotidianas de profissionais de saúde no contexto da implantação da segurança do paciente: entre o prescrito e o real" que contribuiu para um Trabalho de Conclusão de Curso intitulado "A identificação do paciente em unidade de saúde mental", apresentado ao Curso de Graduação em Enfermagem da Universidade Federal de Viçosa (UFV). Viçosa, MG.

Recebido em: 16/07/2019

Aprovado em: 13/12/2019

Endereço de correspondência:

Andréia Guerra Siman

Av. Peter Henry Rolfs, $\mathrm{s} / \mathrm{n}$, Campus Universitário.

CEP: 36570-900 - Viçosa/MG - Brasil

E-mail: ago.80@hotmail.com 